\title{
OPEN The $\sigma^{54}$ system directly regulates bacterial natural product genes
}

\begin{abstract}
Muqing Ma, Roy D. Welch \& Anthony G. Garza
Bacterial-derived polyketide and non-ribosomal peptide natural products are crucial sources of therapeutics and yet little is known about the conditions that favor activation of natural product genes or the regulatory machinery controlling their transcription. Recent findings suggest that the $\sigma^{54}$ system, which includes $\sigma^{54}$-loaded RNA polymerase and transcriptional activators called enhancer binding proteins (EBPs), might be a common regulator of natural product genes. Here, we explored this idea by analyzing a selected group of putative $\sigma^{54}$ promoters identified in Myxococcus xanthus natural product gene clusters. We show that mutations in putative $\sigma^{54}$-RNA polymerase binding regions and in putative Nla28 EBP binding sites dramatically reduce in vivo promoter activities in growing and developing cells. We also show in vivo promoter activities are reduced in a nla28 mutant, that Nla28 binds to wild-type fragments of these promoters in vitro, and that in vitro binding is lost when the Nla28 binding sites are mutated. Together, our results indicate that $M$. xanthus uses $\sigma^{54}$ promoters for transcription of at least some of its natural product genes. Interestingly, the vast majority of experimentally confirmed and putative $\sigma^{54}$ promoters in $M$. xanthus natural product loci are located within genes and not in intergenic sequences.
\end{abstract}

\author{
Abbreviations \\ EBP Enhancer binding protein \\ DBD DNA binding domain \\ PK Polyketide \\ NRP Non-ribosomal peptide \\ UTR Untranslated region
}

The $\sigma^{54}$ regulatory system modulates transcription of a wide variety of bacterial genes. One crucial component of this regulatory system is the $\sigma^{54}$ protein, which directs RNA polymerase to conserved DNA sequences located in the -12 and -24 -bp regions of $\sigma^{54}$ promoter elements ${ }^{1,2}$. Enhancer binding proteins (EBPs), which are transcriptional activators, are also crucial for the normal function of the $\sigma^{54}$ regulatory system. Namely, EBPs are ATPases that use the energy from ATP hydrolysis to help $\sigma^{54}$-RNA polymerase form an open promoter complex and initiate transcription ${ }^{3-5}$. Bacteria typically have one gene for $\sigma^{54}$, but often have multiple genes for EBPs; each EBP works with $\sigma^{54}$ to regulate a subset of $\sigma^{54}$ promoters, which the EBP identifies via specific tandem repeat sequences or enhancer elements ${ }^{6,7}$. Interestingly, the tandem repeat binding sites of EBP dimers are typically located 80- to 150-bp upstream of the -12 and - 24-bp regions of $\sigma^{54}$ promoters; hence, it seems likely that many $\sigma^{54}$ promoters have intrinsically curved DNA sequences or binding sequences for DNA bending proteins, as EBP dimers directly contact $\sigma^{54}$-RNA polymerase ${ }^{8-10}$.

EBPs generally contain three domains: an N-terminal signaling domain, a central ATPase domain that is responsible for ATP hydrolysis and transcriptional activation, and a C-terminal DNA-binding domain (DBD) that recognizes a specific DNA sequence ${ }^{7}$. Typically, the N-terminal signaling domain modulates the ATPase activity of the EBP in response to an intracellular or extracellular signal. In some cases, the N-terminal domain binds directly to a signaling molecule. However, the N-terminal domain of most EBPs is modified (e.g., by phosphorylation) by a signal transduction partner such as a histidine protein kinase that detects the signall ${ }^{11}$. Because $\sigma^{54}$-RNA polymerase requires the energy from EBP-catalyzed ATP hydrolysis to initiate transcription and the EBP's ATPase activity is controlled by signal input, the $\sigma^{54}$ system is able to tightly control transcription of its target genes.

Historically, $\sigma^{54}$ was viewed as specialized regulatory system that was mainly dedicated to transcription of genes involved in nitrogen assimilation or nitrogen fixation ${ }^{12,13}$. In recent years however, it has become clear that the $\sigma^{54}$ system is important for transcription of many types of bacterial genes. For example, the $\sigma^{54}$ system in Bacillus cereus, Pseudomonas aeruginosa and Escherichia coli modulates transcription of genes involved amino acid metabolism, the response to reactive nitrogen species and the phage shock response, respectively ${ }^{14-16}$. In 
Caulobacter crescentus, Pseudomonas aeruginosa, Pseudomonas putida and Vibrio cholerae the $\sigma^{54}$ system regulates genes that are important for flagellar biosynthesis and motility ${ }^{17-21}$, and in Pseudomonas aeruginosa the $\sigma^{54}$ system is implicated in transcription of genes involved in quorum sensing, biofilm formation and virulence $\mathrm{e}^{22-24}$.

The $\sigma^{54}$ system in the soil bacterium Myxococcus xanthus has been studied extensively and has some rare properties. Namely, $M$. xanthus is one of the rare bacterial species in which the $\sigma^{54}$ system has been linked to growth in nutrient rich conditions. For example, inactivation of the nla4 or nla 18 EBP gene severely impairs $M$. xanthus growth in nutrient rich media ${ }^{25-27}$. Presumably, the relatively slow growth of the nla4 mutant and nla18 mutant is due at least in part to changes in the basal levels of the intracellular starvation signal (p)ppGpp and the resulting changes in gene expression.

M. xanthus also has an unusually large repertoire of 53 EBP genes ${ }^{28}$. All of the EBPs were characterized a number of years ago and many of the EBPs were implicated in motility ${ }^{25,29,30}$ and in starvation-induced biofilm formation $^{25,30-36}$, which yields spore-filled aerial structures called fruiting bodies. Six of the EBPs that begin functioning in the early to middle stages of biofilm development, which is also known as fruiting body development, form a regulatory cascade ${ }^{30}$. This EBP cascade is reminiscent of the sigma factor cascade that controls the sequential stages of spore development in Bacillus subtilis ${ }^{37}$, as pairs of EBPs functioning at one stage of development directly activate transcription of an EBP gene important for the next developmental stage.

$\mathrm{Nla} 28$ is one of the early-functioning developmental EBPs that participates in the transcriptional cascade. A putative tandem repeat promoter binding site for Nla28 dimers was identified and analyzed using bioinformatics and experimentation ${ }^{30,38,39}$. The consensus Nla28 binding site $[\mathrm{CT}(\mathrm{C} / \mathrm{G}) \mathrm{CG}(\mathrm{C} / \mathrm{G}) \mathrm{AG}$ consensus half site], which was generated from these studies, was subsequently used to search the $M$. xanthus genome sequence for Nla28 target promoters/genes located outside the EBP cascade ${ }^{28,39}$. A number of these putative Nla28 target promoters were identified in natural product gene clusters. This was an intriguing finding, as Volz et al. previously showed that two M. xanthus EBPs (HsfA and MXAN4899) are capable of binding to fragments of natural product gene promoters ${ }^{40}$. Furthermore, it was previously suggested that the $\sigma^{54}$ system might be a key regulator of polyketide (PK) and non-ribosomal peptide (NRP) natural product genes in M. xanthus, and in bacteria in general, based on bioinformatics ${ }^{38}$

Here, we explored the idea that the $\sigma^{54}$ system modulates expression of M. xanthus natural product genes. We focused on three potential $\sigma^{54}$ promoter targets of Nla28 that are located in $M$. xanthus natural product gene clusters ${ }^{38}$. The activities of the three natural product promoters increase during growth, with peak activities occurring during the transition into stationary phase. The activities of the promoters also increase during starvation-induced development. These findings are consistent previous studies linking expression of bacterial natural product genes to changes in nutritional status ${ }^{41-45}$. Our in vivo and in vitro mutational analyses indicate that three natural product promoters are indeed $\sigma^{54}$ promoter elements and direct targets of Nla28, as predicted via bioinformatics. An examination of the characterized and uncharacterized $\sigma^{54}$ promoters in $M$. xanthus natural product gene clusters yielded a striking result; the vast majority are predicted to be intragenic not intergenic promoters. These results are consistent with previous experimental findings, which placed many characterized M. xanthus $\sigma^{54}$ promoters in intragenic regions ${ }^{30,39}$.

\section{Materials and methods}

Bacterial strains, plasmids and media. Bacterial strains and plasmids used in the study are listed in Table S1. M. xanthus strains were grown at $32{ }^{\circ} \mathrm{C}$ in CTTYE broth [ $1 \%$ Casitone, $0.2 \%$ yeast extract, $10 \mathrm{mM}$ Tris (pH 8.0), $1 \mathrm{mM} \mathrm{KH}_{2} \mathrm{PO}_{4}(\mathrm{pH} 7.6), 8 \mathrm{mM} \mathrm{MgSO}_{4}$ ] or on CTTYE plates containing $1.5 \%$ agar. Fifty $\mu \mathrm{g} / \mathrm{ml}$ of kanamycin or $10 \mu \mathrm{g} / \mathrm{ml}$ of tetracycline were added to CTTYE broth and CTTYE agar plates as needed. CTT soft agar (CTTSA), which is used to plate electroporated $M$. xanthus cells, contains $1 \%$ Casitone, $10 \mathrm{mM}$ Tris ( $\mathrm{pH}$ 8.0), $1.0 \mathrm{mM} \mathrm{KH}_{2} \mathrm{PO}_{4}$ (pH 7.6), 8.0 $\mathrm{mM} \mathrm{MgSO}_{4}$, and $0.7 \%$ agar. Submerged culture development of M. xanthus strains occurred in 24-well polystyrene plates containing $100 \mu \mathrm{l}$ of MC7 buffer [10 mM morpholinepropanesulfonic acid (MOPS; pH 7.0), $1 \mathrm{mM} \mathrm{CaCl}_{2}$ ]. Unless otherwise stated, E. coli strains were grown in Luria-Bertani (LB) broth $[0.5 \%$ yeast extract, $1 \%$ tryptone, $1 \% \mathrm{NaCl}$ ) or on $\mathrm{LB}$ plates containing $1.5 \%$ agar. LB broth and $\mathrm{LB}$ plates were supplemented with $100 \mu \mathrm{g} / \mathrm{ml}$ of ampicillin, $50 \mu \mathrm{g} / \mathrm{ml}$ of kanamycin or $10 \mu \mathrm{g} / \mathrm{ml}$ of tetracycline as needed. For Nla28-DBD expression, E. coli strains were grown in rich LB broth $[0.5 \%$ yeast extract, $1 \%$ tryptone, $0.5 \% \mathrm{NaCl}, 0.2 \%$ glucose] supplemented with $100 \mu \mathrm{g} / \mathrm{ml}$ of ampicillin.

M. xanthus growth and development. M. xanthus strains were grown by inoculating cells into flasks containing CTTYE broth and incubating the cultures at $32^{\circ} \mathrm{C}$ with vigorous swirling. Development was induced as previously described ${ }^{25}$. Briefly, M. xanthus cells were grown in CTTYE broth until the cultures reached a density of approximately $5 \times 10^{8}$ cells $/ \mathrm{ml}$, the cells were pelleted, the supernatant was removed, and the cells were resuspended in MC7 buffer to a density of $5 \times 10^{9} \mathrm{cells} / \mathrm{ml}$. Forty $\mu$ l aliquots of the cell suspensions were placed into polystyrene plate wells containing $100 \mu \mathrm{l}$ of MC7 buffer and the polystyrene plates were transferred to a $32{ }^{\circ} \mathrm{C}$ incubator for $24 \mathrm{~h}$.

Standard DNA procedures. Chromosomal DNA from wild-type M. xanthus strain DK1622 $2^{46}$ was extracted using ZYMO Research gDNA extraction kit. Oligonucleotides used in PCR reactions were synthesized by Integrated DNA Technologies (IDT) and are listed in Table S2. Plasmid DNA was extracted using the Promega Nucleic acid purification kit. Amplified and digested DNA fragments were purified using the Gel Extraction Minipreps kit of Bio Basic. For all kits, the manufacturer's protocols were used. The compositions of all plasmids and promoter fragments were confirmed by DNA sequencing (Genewiz). 
Site-directed mutations. Site-directed mutations in putative $\sigma^{54}$ promoter elements were generated using the Quick Lightning Mutagenesis Kit from Agilent Technologies and the manufacturer's protocol. Briefly, promoter fragments containing the putative $\sigma^{54}$-RNA polymerase binding site in the -12 and - 24-bp regions and the upstream Nla28 tandem repeat binding site were cloned into pCR 2.1 TOPO vector (Invitrogen). Mutations in the - 12-bp region, the - 24-bp region, the spacer between the - 12 and - 24-bp regions were generated using primers carrying the appropriate nucleotide changes (Table S2), plasmids containing the promoter fragments and PfuUltra DNA polymerase. Parental plasmid DNA was removed by digesting with DpnI and transformed into E. coli for conversion into duplex form. Plasmid-borne promoter mutations were verified by DNA sequence analysis. Promoter fragments carrying Nla28 binding site mutations $\left(\mathrm{P}_{\mathrm{EM} 1286}, \mathrm{P}_{\mathrm{EM} 1579}\right.$ and $\left.\mathrm{P}_{\mathrm{EM} 3778}\right)$ were synthesized by IDT; the first putative Nla28 half binding site in each promoter was changed to AAAAAAAA. The mutant promoter fragments were then subcloned into the promoterless lac $Z$ expression vector pREG1727 ${ }^{47}$, introduced into M. xanthus strains and analyzed as described below.

In vivo analysis of wild-type and mutant promoters. Wild-type and mutant MXAN1286, MXAN1579, MXAN1603 and MXAN3778 promoter fragments were cloned into the promoterless lacZ expression vector pREG1727 to create lac $Z$ transcriptional fusions ${ }^{47}$. The plasmids were introduced into strain DK1622 or a derivative of strain DK1622 carrying an insertion in the nla28 gene, and cells carrying a plasmid integrated at the Mx8 phage attachment site in the chromosome were identified via PCR. The in vivo activities of wild-type and mutant promoters were determined by measuring the specific activities of $\beta$-galactosidase in cells developing in submerged cultures for $1,2,6,12$ or $24 \mathrm{~h}$, or growing in CTTYE broth for various amounts of time $\mathrm{e}^{48,49}$. Mean wild-type and mutant promoter activities were compared using a two-way analysis of variance (ANOVA) and Tukey's multiple comparisons post hoc tests, considering two independent variables in each group. The significance level was set at $\mathrm{p}<0.05$ or lower. GraphPad Prism software v8.4 (GraphPad Software, La Jolla, CA, USA) was used for all analyses.

Expression and purification of Nla28-DBD. A fragment of the nla28 gene corresponding to the Nla28 DNA binding domain (Nla28-DBD) ${ }^{30}$ was PCR amplified using gene-specific primers (Table S2), and then cloned into the pMAL-c5x vector. The resulting plasmid, which creates an N-terminal Maltose Binding Protein (MBP) fusion to Nla28-DBD, was introduced into E. coli strain BL21 (DE3) using chemical transformation. Cells containing the Nla28-DBD expression plasmids were grown in rich LB broth to a density of $2 \times 10^{8}$ cells/ $\mathrm{ml}$. Protein expression was induced by the addition of $0.3 \mathrm{mM}$ IPTG to the culture and the subsequent incubation of the culture for $12 \mathrm{~h}$ at $15^{\circ} \mathrm{C}$. Cells were pelleted via centrifugation and resuspended in $25 \mathrm{ml}$ column buffer (20 mM Tris- $\mathrm{HCl}, 200 \mathrm{mM} \mathrm{NaCl}, 1 \mathrm{mM}$ EDTA, $5 \mathrm{U} / \mathrm{ml}$ DNase I) per liter of culture. The resuspended cells were lysed by a combination of freeze-thawing and sonication, and pelleted by centrifugation. The crude extract (supernatant) containing Nla28-DBD was diluted by adding $125 \mathrm{ml}$ of cold column buffer to every $25 \mathrm{ml}$ aliquot of crude extract. For purification of Nla28-DBD from the diluted crude extract, $15 \mathrm{ml}$ of amylose resin was placed in a $2.5 \times 10 \mathrm{~cm}$ column and the amylose resin was washed with $250 \mathrm{ml}$ cold column buffer. Diluted crude extract containing Nla28-DBD was loaded onto amylose columns at a flow rate of $5 \mathrm{ml} / \mathrm{min}$ and washed with $600 \mathrm{ml}$ cold column buffer at a flow rate of $10 \mathrm{ml} / \mathrm{min}$. Nla28-DBD was eluted using $100 \mathrm{ml}$ cold column buffer containing $10 \mathrm{mM}$ maltose; the flow rate was $5 \mathrm{ml} / \mathrm{min}$ and 20 fractions containing $5 \mathrm{ml}$ were collected. The presence of eluted Nla28-DBD was detected by UV absorbance at $280 \mathrm{~nm}$. Nla28-DBD- containing fractions were pooled and incubated with $1 \mathrm{mg}$ of Factor Xa at $4{ }^{\circ} \mathrm{C}$ overnight to cleave the MBP tag. Nla28-DBD was separated from MBP and concentrated to about $1 \mathrm{mg} / \mathrm{ml}$ using Amicon Ultra centrifugal filter units (EMD Millipore). SDS-PAGE and Bradford assays were used to determinate the purity and concentration of Nla28-DBD.

Electrophoretic mobility shift assays (EMSAs). Purified Nla28-DBD was expected to bind to wild-type MXAN1286, MXAN1579 and MXAN3778 promoter fragments carrying a putative Nla28 tandem repeat binding site. Using the 5' Cy5-labelled oligonucleotides shown in Table S2, Cy5-labelled MXAN1286, MXAN1579 and MXAN3778 promoter fragments $\left(\mathrm{Cy} 5-\mathrm{P}_{1286}, \mathrm{Cy} 5-\mathrm{P}_{1579}\right.$ and Cy5- $\left.\mathrm{P}_{3778}\right)$ were generated via PCR; each promoter fragment contained a putative wild-type Nla28 binding site. Three mutant derivatives of these $5^{\prime}$ Cy5-labelled promoter fragments (Cy5- $\mathrm{P}_{\text {mut1286 }}$, Cy5- $\mathrm{P}_{\text {mut1579 }}$ and Cy5- $\left.\mathrm{P}_{\text {mut3778 }}\right)$ were synthesized; the first putative Nla28 half binding site in each promoter was changed to AAAAAAAA. All PCR-generated and synthesized promoter fragments were gel-purified and used in subsequent EMSAs. In EMSA reactions, $2 \mu \mathrm{M}$ purified Nla28DBD was incubated with $1 \mathrm{ng}$ of 5' Cy5-labelled wild-type promoter fragment (Cy5- $\mathrm{P}_{1286}, \mathrm{Cy} 5-\mathrm{P}_{1579}$ and Cy5$\left.\mathrm{P}_{3778}\right)$ or 5' Cy5-labelled mutant promoter fragment (Cy5- $\mathrm{P}_{\text {mut1286}}$, Cy5- $\mathrm{P}_{\text {mut1579 }}$ and Cy5- $\left.\mathrm{P}_{\text {mut3778 }}\right)$ in EMSA buffer (25 mM Tris acetate, $8.0 \mathrm{mM}$ magnesium acetate, $10 \mathrm{mM} \mathrm{KCl}, 1 \mathrm{mM} \mathrm{DTT}, \mathrm{pH}$ 8.0) for $30 \mathrm{~min}$ at $30^{\circ} \mathrm{C}$. The samples were then analyzed using PAGE under non-denaturing conditions and imaged using a Bio-Rad imager.

\section{Results}

Identifying putative $\sigma^{54}$ promoter elements in $M$. xanthus natural product gene clusters. In a previous study ${ }^{38}$, the algorithm developed by Studholme et al. ${ }^{50}$ was used to examine whether the $\sigma^{54}$ system might be a common regulator of bacterial natural product genes. Namely, 180 annotated PK and NRP gene clusters from 58 bacterial species were analyzed for sequences that closely match the $\sigma^{54}$ promoter consensus in the - 12-bp region and in the -24 -bp region (i.e., the regions of $\sigma^{54}$-RNA polymerase binding). The results, which uncovered 124 clusters with at least one $\sigma^{54}$ promoter based on consensus matching, supported the idea that the $\sigma^{54}$ system might be a general regulator of bacterial natural product genes.

The goal of the work presented here was to examine whether a major producer of bacterial natural products (M. xanthus) uses the $\sigma^{54}$ system for transcription of PK and NRP gene clusters, as predicted in the bioinformatics 
A

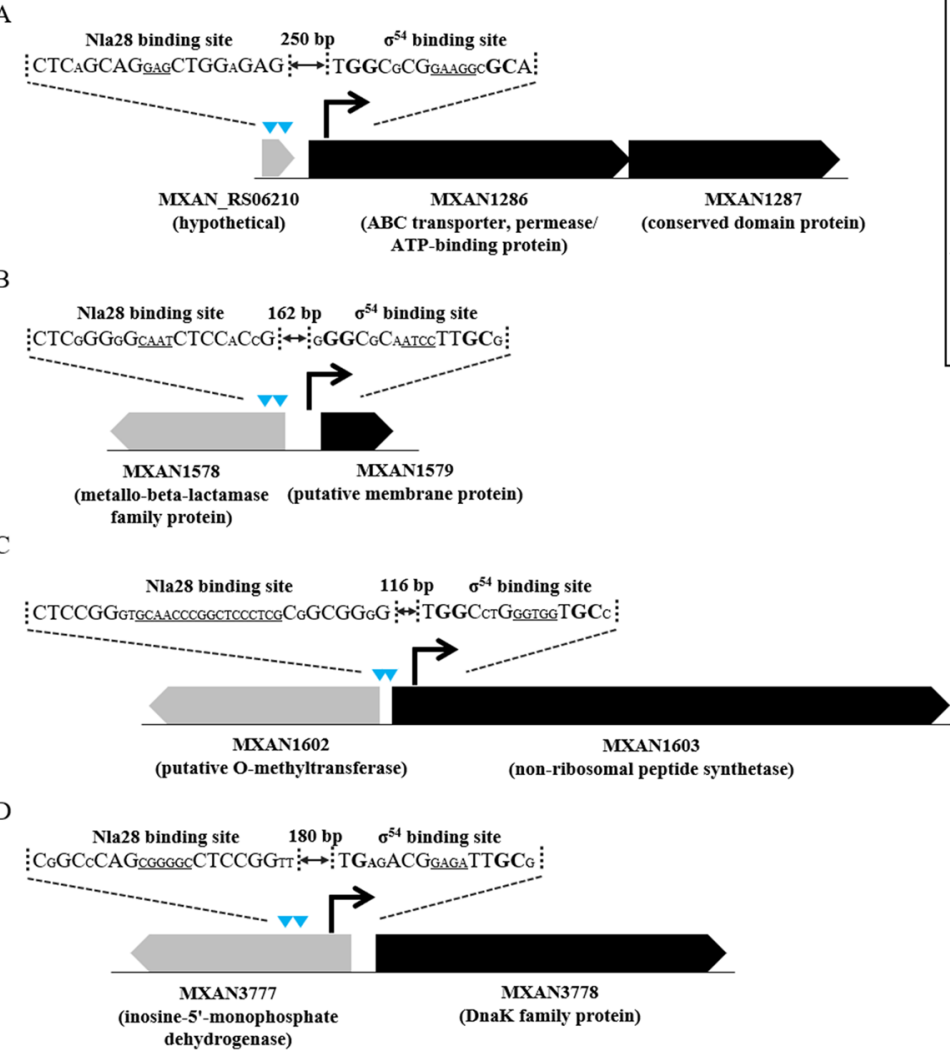

Figure 1. The promoter regions of the MXAN1286, MXAN1579, MXAN1603 and MXAN3778 natural product loci. Nucleotides the match those in the consensus Nla28 binding site or the consensus $\sigma^{54}$ RNA polymerase binding site are relatively large. The conserved GC dinucleotide in - 12-bp region and the conserved GG dinucleotide in - 24-bp region of the putative $\sigma^{54}$ RNA polymerase binding sites are in bold. The underlined nucleotides represent the spacers between the two half Nla28 binding sites or the spacers between -12 and - 24-bp promoter regions.

analysis of Stevens et al. ${ }^{38}$. We focused on the putative $\sigma^{54}$ promoters upstream of MXAN1286, MXAN1579, MXAN1603 and MXAN3778, as these genes were linked to natural product biosynthetic genes/clusters. Namely, the MXAN1286 gene is embedded in a putative NRP gene cluster and flanked by genes that are likely to be involved in NRP biosynthesis. MXAN1579 and MXAN1603 are predicted to be part of another M. xanthus NRP gene cluster. MXAN1603 is a putative non-ribosomal peptide synthetase gene. MXAN1579 is embedded in the cluster and flanked by genes such as MXAN1603 that are likely to be involved in NRP biosynthesis. MXAN3778 is adjacent to a putative hybrid non-ribosomal peptide synthetase/type I polyketide synthase gene and possibly part of the same operon ${ }^{38}$.

The MXAN1286, MXAN1579, MXAN1603 and MXAN3778 genes were also identified as potential targets of the EBP Nla2 $8^{30,39}$; 8-bp repeat sequences, which are close matches to the consensus Nla28 half binding site, were identified upstream of the putative -12 and -24 -bp regions (Fig. 1). It is notable that six of the eight putative Nla28 half binding sites and three of the four putative $\sigma^{54}$-RNA polymerase binding sites are located within protein coding sequences. Indeed, residence in an intragenic region is common among the putative PK and NRP $\sigma^{54}$ promoters identified in the M. xanthus genome (Fig. 2, Table S3 and Figure S1), and among the $\sigma^{54}$ promoters known to be regulated by the EBP Nla $28^{30,39}$. It is also noteworthy that many of the putative $\sigma^{54}$ promoter elements are located within operons; they might serve as internal promoters (Fig. 2, Table S3 and Figure S1).

Mutations in the putative - 12-bp region, - 24-bp region or spacer region impair the in vivo activities of natural product promoters. $\sigma^{54}$ promoters typically have a GC dinucleotide in the -12 -bp region and a GG dinucleotide in the -24 -bp region ${ }^{1,2}$. These dinucleotides and the 4-bp spacer between the -12 and - 24-bp regions are often referred to as the hallmarks of $\sigma^{54}$ promoters. Indeed, the putative $\sigma^{54}$ promoters in the MXAN1286, MXAN1579 and MXAN1603 natural product loci appear to have these hallmarks (Fig. 1). As for the $\sigma^{54}$ promoter identified in the MXAN3778 locus, one hallmark variation is apparent. Namely, the - 24-bp region has GA instead of a GG dinucleotide. Despite this variation in - 24-bp region dinucleotide, MXAN3778 was classified as a potential $\sigma^{54}$ promoter, as a 1-bp change in either the GC or GG dinucleotide has been identified in a number of characterized $\sigma^{54}$ promoters, including the $\sigma^{54}$ promoters in the M. xanthus asgE, spi and nla6 loci ${ }^{30,51-53}$.

To confirm that the natural product loci have bona fide $\sigma^{54}$ promoter elements, we analyzed the putative $\sigma^{54}$ promoter hallmarks via mutational analysis. In particular, a 446-bp DNA fragment of the MXAN1286 promoter 


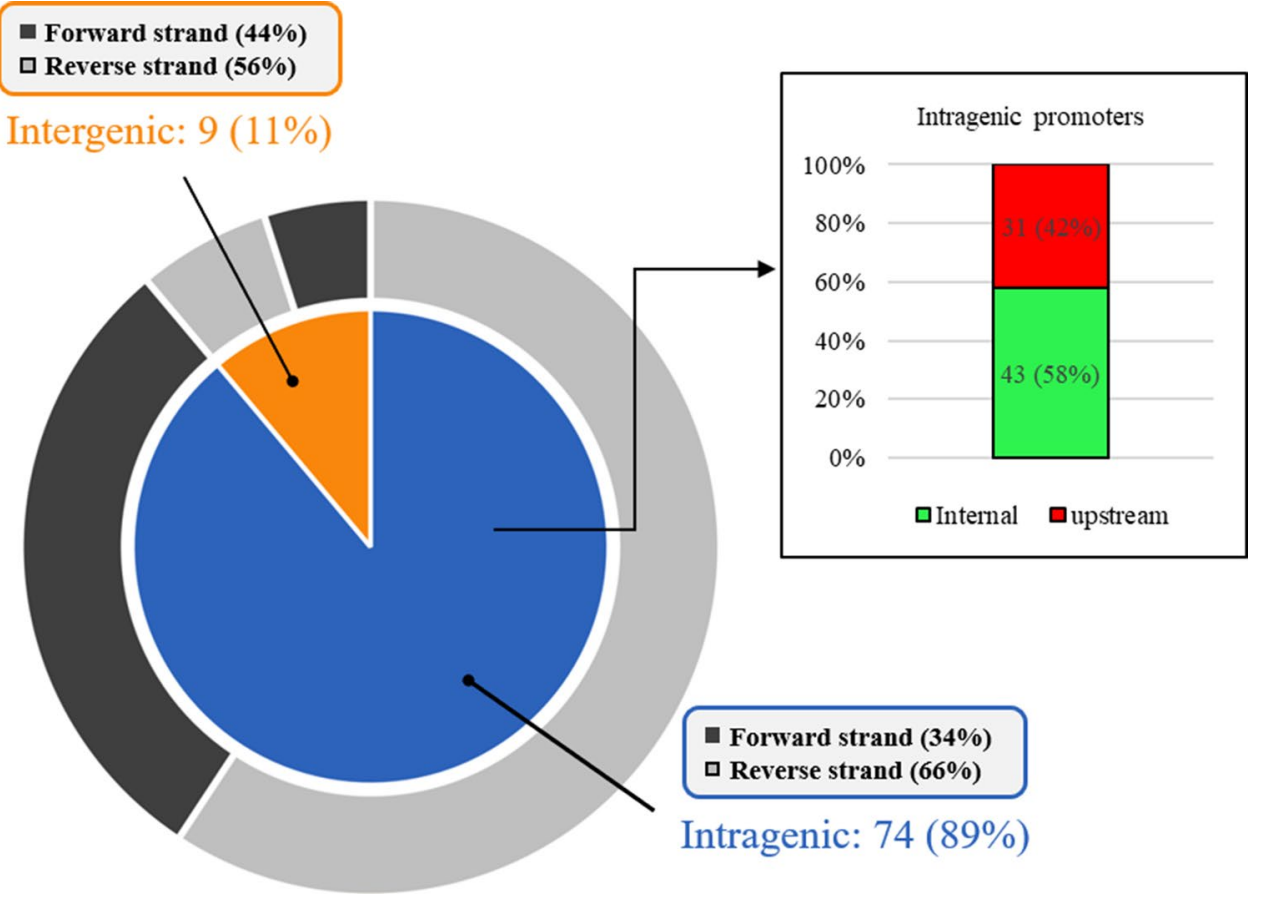

Figure 2. Locations of putative PK/NRP $\sigma^{54}$ promoters identified in the $M$. xanthus genome. Of the 83 putative $\mathrm{PK} / \mathrm{NRP} \sigma^{54}$ promoters identified in $M$. xanthus genome sequence, $74(89 \%)$ are located in protein coding sequences (intragenic promoters) and $9(11 \%)$ are located in non-coding sequences (intergenic promoters). Of the 74 intragenic promoters, 43 are located within a protein coding sequence in an operon or within the protein coding sequence of a single gene (internal promoters), and 31 are located in the protein coding sequence of an upstream gene (upstream promoters).

region, a 402-bp fragment of the MXAN1579 promoter region, a 518 bp fragment of the MXAN1603 promoter region and a 600-bp fragment of the MXAN3778 promoter region were used to generate mutations in the putative - 12-bp, - 24-bp and spacer regions. We should note that the MXAN1286 promoter fragment contains both of the putative $\sigma^{54}$ promoters located in the $5^{\prime}$ end of the MXAN1286 gene and the MXAN1579 promoter fragment contains the two putative intergenic $\sigma^{54}$ promoters that are oriented in the appropriate direction with respect to MXAN1579 transcription (Table S3 and Figure S1). However, we focused the mutational analysis on the putative $\sigma^{54}$ promoter that is the farthest downstream in the MXAN1286 coding sequence (+111 bp; see Table S3) and the putative $\sigma^{54}$ promoter that is farthest upstream of the predicted transcriptional start site of MXAN1579 gene ( -104 bp; see Table S3) after considering a number of factors, including the presence of $\sigma^{54}$ promoter hallmarks, the overall match to the $\sigma^{54}$ promoter consensus sequence [TGGCACG-4 N-TTGC(T/A)] and the distance from putative Nla28 binding sites ${ }^{1,2}$. The mutations that we generated are the following: the GC dinucleotide in the - 12-bp region was replaced with a TT, the GG (GA in MXAN3778) dinucleotide in the-24-bp region was replaced with a TT, or $1 \mathrm{bp}$ in the spacer between the - 12 and -24-bp regions was deleted. Subsequently, wildtype and mutant promoter fragments were fused to the promoterless lacZ gene in plasmid pREG $1727^{47}$ and the lac $Z$ transcriptional fusion plasmids were introduced into wild-type $M$. xanthus strain DK1622 (the plasmids integrated at the Mx8 phage attachment site in the chromosome).

Wild-type and mutant promoter activities during growth in CTTYE broth and fruiting body development in MC7 starvation buffer were inferred from the levels of lacZ expression. As shown in Fig. 3A-C, the in vivo activities of the MXAN1286, MXAN1579 and MXAN3778 promoters increased about 4.6-fold, 1.8-fold and twofold, respectively, during growth in CTTYE broth. It is notable that peak levels of promoter activity occurred at the highest cell densities, which correspond to the transition into stationary phase and presumably nutrient depletion. This of course agrees with the data shown in Fig. 3D-F, which revealed a 1.8- to 2.5-fold increase in the vivo activities of the three promoters during development in MC7 starvation buffer. Mutations in the - 12-bp region, the -24 -bp region and spacer dramatically reduced (about 3.2- to 11.1-fold) the activities of these promoters at all cell densities during growth and time points in development. Thus, mutations in the putative sites of $\sigma^{54-}$ RNA polymerase binding substantially impacted the activities of the MXAN1286, MXAN1579 and MXAN3778 promoters in growing and developing cells, supporting the prediction that the three natural product loci use $\sigma^{54}$ promoter elements for transcription.

In contrast to the other promoters, the MXAN1603 promoter only showed a slight increase (about 1.3-fold) in activity during growth in CTTYE (Figure S2A) and during development in MC7 starvation buffer (Figure S2B). Furthermore, mutations in the -12 -bp region, the -24 -bp region and the spacer caused a modest, but statistically significant decrease $(\mathrm{P}<0.001)$ in promoter activity at all cell densities during growth and after $1 \mathrm{~h}$ of 
A

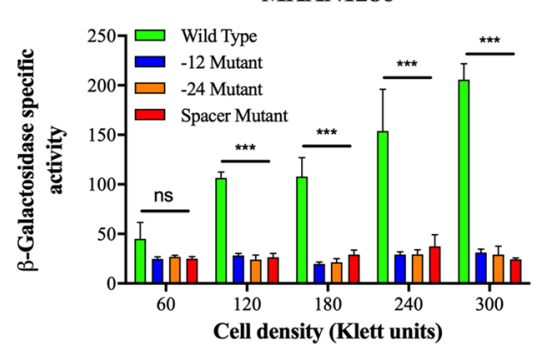

$\mathrm{D}$

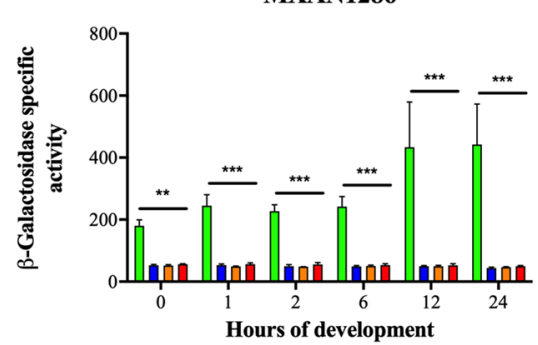

B

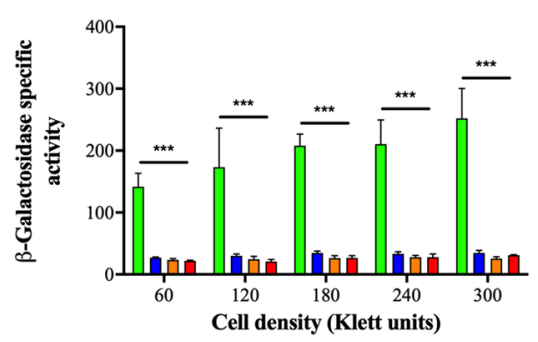

$\mathrm{E}$

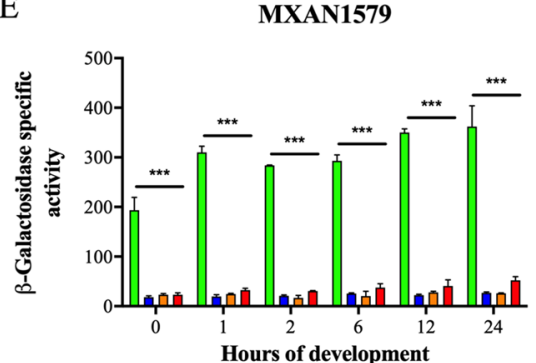

$\mathrm{C}$

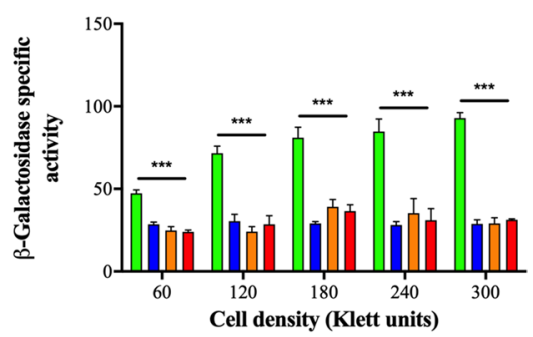

$\mathrm{F}$

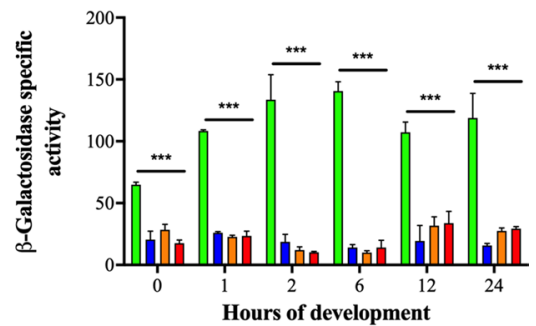

Figure 3. In vivo activities of wild-type MXAN1286, MXAN1579 and MXAN3778 promoters and derivatives of the promoters carrying a mutation in the putative - 12-bp region, - 24-bp region or spacer region. Wildtype and mutant fragments of the MXAN1286, MXAN1579 and MXAN3778 promoters were cloned into a lacZ expression vector and transferred to the wild-type M. xanthus strain DK1622. At various cell densities during growth $(\mathbf{A}-\mathbf{C})$ and time points during development $(\mathbf{D}-\mathbf{F})$, $\beta$-galactosidase-specific activities (defined as nanomoles of ONP produced per minute per milligram of protein) in cells carrying a wild-type or a mutant promoter fragment were determined. $(\mathrm{N}=3$ at each density or time point; error bars are standard deviations of the means; ${ }^{* * *} \mathrm{p}<0.001 ;{ }^{* *} \mathrm{p}<0.01 ;{ }^{*} \mathrm{p}<0.05$ for in vivo activities of mutant promoters versus wild-type promoters).

development (Figure S2). Thus, mutations in the putative $\sigma^{54}$-RNA polymerase binding site in the MXAN1603 promoter region only had a modest impact on growth-related and developmental activities. Our interpretation of this result is that the fragment of the MXAN1603 promoter region contains an unidentified promoter element that is responsible for the majority of the observed development and growth-related activities and that the putative $\sigma^{54}$-type promoter element only makes a minor contribution to the observed activities.

The in vivo activities of natural product promoters are impacted by inactivation of the nla28 gene. EBPs are essential for transcription at $\sigma^{54}$ promoters, as EBP-mediated ATP hydrolysis opens the $\sigma^{54}$-RNA polymerase promoter complex so that transcription can initiate ${ }^{3-5}$. Since the $\sigma^{54}$ promoters in the MXAN1286, MXAN1579, and MXAN3778 loci were identified as potential targets of Nla28, we determined whether the activities of wild-type promoter fragments are reduced as predicted in a mutant containing an inactivated nla28 gene ${ }^{25}$ (Note that the MXAN1603 $\sigma^{54}$ promoter was not analyzed further because it is unlikely to be the primary promoter used during growth or development). MXAN1286, MXAN1579, and MXAN3778 promoter activities in wild-type and nla28 mutant cells grown in CTTYE broth are shown in Fig. 4A-C. As predicted, inactivation of nla28 abolished the growth phase regulation of all three promoters and caused about 3.1to fourfold reduction in peak promoter activities at the highest cell densities. Inactivation of nla28 also abolished the developmental activities of the promoters, as the promoters did not show the typical increases in activities when nla 28 cells were placed in MC7 starvation buffer (Fig. 4D-F). Indeed, the peak developmental promoter activities in nla 28 mutant cells were reduced about 3.4 - to 5.0 -fold relative to the corresponding peak activities in wild-type cells. These findings indicate that Nla28 is crucial for the observed growth-related and developmental activities of the MXAN1286, MXAN1579, and MXAN3778 natural product promoters.

Mutations in putative Nla28 half sites impact the in vivo activities of natural product promoters. As noted above, we identified 8-bp repeat sequences, which are close matches to the consensus Nla28 half binding site $[\mathrm{CT}(\mathrm{C} / \mathrm{G}) \mathrm{CG}(\mathrm{C} / \mathrm{G}) \mathrm{AG}]$, in the $\sigma^{54}$ promoters under study here (see Fig. 1). To examine whether the $\sigma^{54}$ promoters are directly regulated by Nla2 8 and to further confirm that the promoters are members of the $\sigma^{54}$ family, mutations were generated in the putative Nla28 binding sites in the MXAN1286, MXAN1579, and MXAN3778 promoter fragments noted above. Namely, the distal (relative to the - 12 and - 24-bp regions) Nla28 half binding site in each promoter fragment was converted to all A nucleotides. Wild-type and mutant promoters were introduced into wild-type strain DK1622 and promoter activities during growth in CTTYE broth and development in in MC7 starvation buffer were determined (Fig. 4). The data revealed that Nla28 binding site mutations abolish the growth phase regulation of all three promoters (Fig. 4A-C). Indeed, the peak mutant promoter activities, which were observed at the highest cell density, were reduced about 2.6- to fivefold compared to 
A

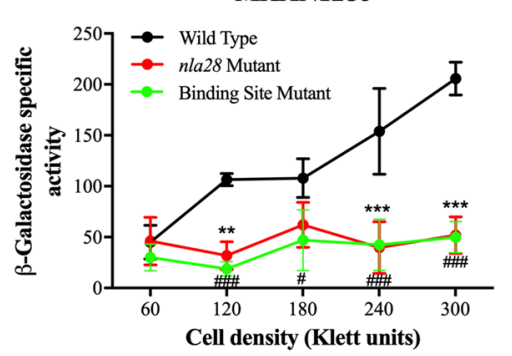

$\mathrm{D}$

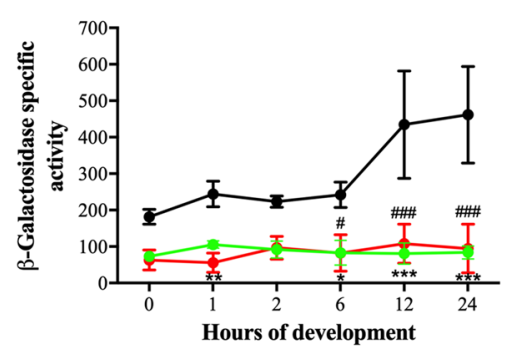

B

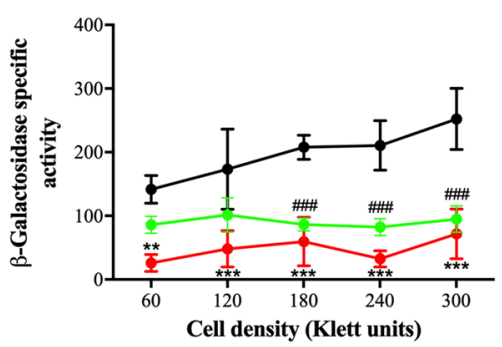

$\mathrm{E}$

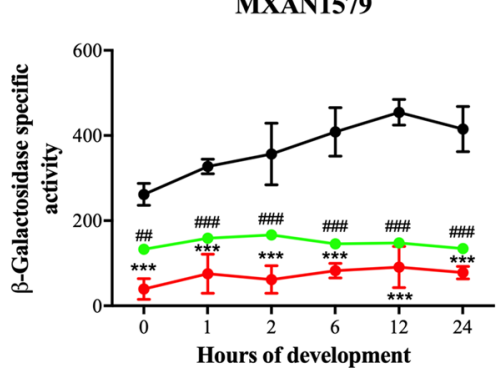

C

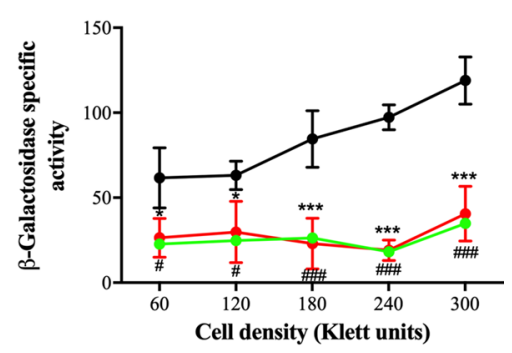

F

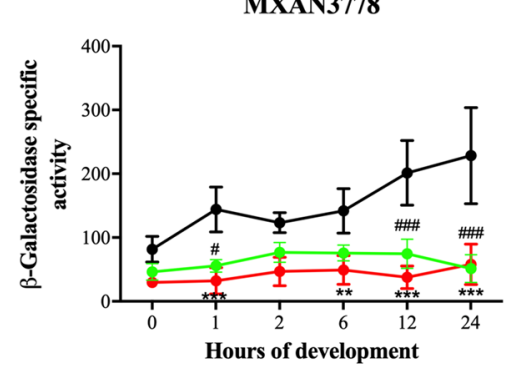

Figure 4. In vivo activities of MXAN1286, MXAN1579 and MXAN3778 promoters in nla28 mutant cells and when Nla28 binding sites are mutated. Wild-type fragments of the MXAN1286, MXAN1579 and MXAN3778 promoters were cloned into a lacZ expression vector and transferred to the wild-type M. xanthus strain DK1622 (Wild Type) or to a derivative of strain DK1622 with an inactivated nla28 gene (nla28 Mutant). In addition, fragments of the MXAN1286, MXAN1579 and MXAN3778 promoters containing one mutated Nla28 half binding site were cloned into a lacZ expression vector and transferred to strain DK1622 (Binding Site Mutant). At various cell densities during growth $(\mathbf{A}-\mathbf{C})$ and time points during development $(\mathbf{D}-\mathbf{F}), \beta$-galactosidasespecific activities in cells carrying a wild-type or a mutant promoter fragment were determined. $(\mathrm{N}=3$ at each density or time point; error bars are standard deviations of the means; ${ }^{* *} \mathrm{p}<0.001 ;{ }^{* *} \mathrm{p}<0.01 ;{ }^{*} \mathrm{p}<0.05$ for in vivo activities of wild-type promoters in nla28 mutant versus in wild-type cells; ${ }^{\# \# \#} \mathrm{p}<0.001 ;{ }^{\# \#} \mathrm{p}<0.01 ;{ }^{\#} \mathrm{p}<$ 0.05 for in vivo activities of binding site mutant promoters versus wild-type promoters in wild-type cells).

that of the corresponding wild-type promoter. Similarly, wild-type MXAN1286, MXAN1579, and MXAN3778 promoters showed increased activities during development and the Nla28 binding site mutations abolished this developmental regulation (Fig. 4D-F). Furthermore, the peak developmental activities of the mutant promoters were reduced from about 2.8- to 4.8 -fold. These findings are consistent with the idea that Nla28 directly regulates the MXAN1286, MXAN1579, and MXAN3778 $\sigma^{54}$ promoters, that the 8-bp repeats that we identified are Nla28 binding sites and that Nla28 is crucial for growth-related and developmental promoter activities.

In vitro analyses indicate that purified Nla28-DBD binds to natural product promoter fragments carrying a wild-type Nla28 binding site, but not to fragments carrying a mutated Nla28 binding site. Electrophoretic mobility shift assays (EMSAs) were used to confirm that the MXAN1286, MXAN1579, and MXAN3778 natural product promoters are targets of the Nla28 EBP. In particular, we used EMSAs to determine whether the purified DNA binding domain of Nla28 (Nla28-DBD) is capable of binding a fragment of the MXAN1286 promoter, MXAN1579 promoter, and MXAN3778 promoter. Each promoter fragment, which corresponded to DNA upstream of - 12 and - 24-bp regions, contained a putative binding site for a Nla28 dimer. As shown in Fig. 5, Nla28-DBD is capable of binding to a MXAN1286, MXAN1579 and MXAN3778 promoter fragment that has a wild-type Nla28 binding site. However, when the distal Nla28 half binding site in each promoter fragment was converted to all A nucleotides, no Nla28-DBD binding was detected (Fig. 5). These findings provide further support that the Nla28 EBP directly regulates the $\sigma^{54}$ promoter elements of the MXAN1286, MXAN1579 and MXAN3778 natural product loci and that the tandem repeats that we identified in the $\sigma^{54}$ promoter elements are Nla28 binding sites.

\section{Discussion}

For decades, bacterial-derived PK and NRP natural products have been a crucial source of therapeutic agents such as antibiotics and yet little information about the regulation of these genes has been uncovered. In a notable study in 2012, Volz et al. showed that two M. xanthus EBPs (HsfA and MXAN4899) are capable of binding to fragments of natural product gene promoters ${ }^{40}$. With this information and the preliminary data from Nla28 studies in mind, Stevens et al. asked if the $\sigma^{54}$ system might be a common regulator natural product genes ${ }^{38}$. Namely, a bioinformatics analysis was used to search for putative $\sigma^{54}$ promoters in the sequences of $180 \mathrm{PK}$ or NRP gene clusters from 58 bacterial species. The results, which revealed that about $70 \%$ of natural product 


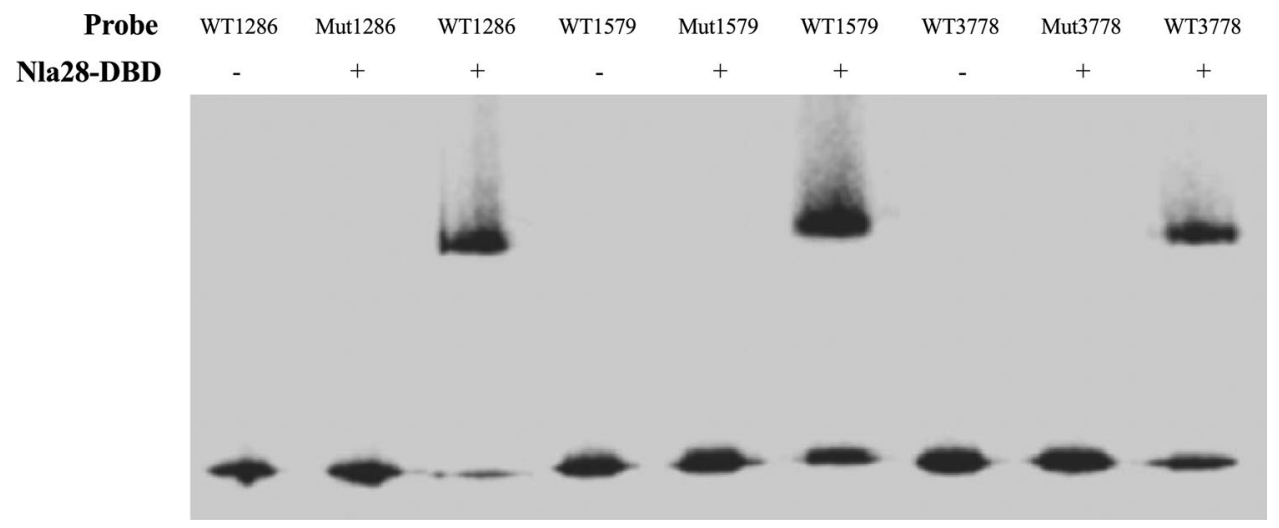

Figure 5. EMSAs performed with Nla28-DBD and a MXAN1286, MXAN1579 or MXAN3778 promoter fragment carrying a wild-type or mutated Nla28 binding site. Binding reactions were performed with $(+)$ or without (-) $2 \mu \mathrm{M}$ of purified Nla28-DBD and a Cy5 end-labeled promoter fragment containing a wild-type (WT) or mutated (Mut) Nla28 binding site.

gene clusters have at least one putative $\sigma^{54}$ promoter, suggested that the $\sigma^{54}$ system might indeed be a common regulator of natural product genes.

One of the goals of this study was to analyze the bioinformatics data experimentally and the putative natural product promoter targets of Nla28 seemed particularly well suited for such a study, given our knowledge of Nla28-mediated regulation. Furthermore, $M$. xanthus is an excellent system to study natural product gene regulation, as this bacterium is a major producer of PKs and NRPs and over 80 putative $\sigma^{54}$ promoters were identified in the PK and NRP gene clusters of strain DK162238 (Table S3 and Figure S1).

Our in vivo and in vitro data indicate that three of the $M$. xanthus natural product promoter regions characterized here (MXAN1579, MXAN3778 and MXAN1286) contain bona fide $\sigma^{54}$ promoter elements and that the $\sigma^{54}$ promoters are targets of the EBP Nla28. Our results also indicate that the $\sigma^{54}$ promoters are crucial for both growth-related and developmental activities. We propose that the fourth natural product promoter region that we characterized, MXAN1603, also contains a $\sigma^{54}$ promoter. However, it seems likely that the MXAN1603 promoter region contains an unidentified promoter element that is responsible for the majority of the observed developmental and growth-related activities and the $\sigma^{54}$-type promoter only makes a minor contribution to these activities.

Previous work indicated that the Nla28 EBP is a response regulator that forms a two component signal transduction system with the membrane-bound sensor histidine kinase Nla28 $\mathrm{S}^{54,55}$. Nla28 begins modulating gene expression in the early stages of starvation-induced fruiting body development ${ }^{25,30,39}$, which led to the suggestion that the Nla28/Nla28S signal transduction system might be a general regulator of starvation-induced or stress-responsive genes ${ }^{55}$. The findings presented here are consistent with this idea. In particular, the MXAN1286, MXAN1579, and MXAN3778 $\sigma^{54}$ promoters are induced in the early-middle stages of fruiting body development and the developmental activities of these promoters are dependent on Nla28 (Fig. 4D-F). The activities of the three promoters also increase during growth, with peaks corresponding to the transition into stationary phase and presumably nutrient depletion. As shown in Fig. 4A-C, this growth-phase regulation is abolished when Nla28 is absent, further linking the Nla28/Nla28S signal transduction system to the starvation response.

The above results point to nutrient depletion/starvation as the potential trigger for Nla28/Nla28S-mediated activation of the MXAN1286, MXAN1579 and MXAN3778 promoters. We suggest that the stringent response and accumulation of (p)ppGpp might also be a key inducing factor, as accumulation of this starvation signal is known to initiate $M$. xanthus fruiting body development ${ }^{56,57}$. Furthermore, it is reasonable to speculate that (p) ppGpp accumulates when $M$. xanthus transitions from exponential growth to stationary phase, since this seems to be the case in other bacterial species ${ }^{58}$. In addition, bacterial natural product gene expression was previously linked to (p)ppGpp accumulation ${ }^{59}$ and stringent response-associated genes such as relA and spoT have been linked to synthesis of bacterial natural products ${ }^{41,42,60}$.

It is interesting that three of the four natural product $\sigma^{54}$ promoters that we characterized are located within the coding sequences of genes (intragenic) and not in intergenic regions (Fig. 1). In the MXAN1286 and MXAN1603 loci, the $\sigma^{54}$ promoters are located in the coding sequence of the first gene of an operon and in the $5^{\prime}$ end of a single gene, respectively. In the other case (MXAN3778), the $\sigma^{54}$ promoter is located in the coding sequence of an upstream gene (Fig. 1). With the exception of MXAN1603, the Nla28 binding sites of the natural product promoters are also intragenic, located in the coding sequence of an upstream gene (Fig. 1). These findings are counter to the commonly held view that bacterial promoter elements are typically located in intergenic regions $s^{61-63}$, but are supported by additional pieces of bioinformatic and experimental data. First, the vast majority of the putative natural product $\sigma^{54}$ promoters shown in Figure S1 and listed in Table S3 are intragenic. Secondly, the majority of the characterized $\sigma^{54}$ promoter targets of the M. xanthus EBP Nla6 are intragenic, located in the coding sequence of an upstream gene ${ }^{53}$. Thirdly, the vast majority of the developmental $\sigma^{54}$ promoter targets of Nla28, which we recently characterized, are located in the coding sequence of an upstream gene or appear to be internal promoters in operons ${ }^{30,39}$. Lastly, genomic-wide studies in Escherichia coli and Salmonella enterica 
serovar Typhimurium uncovered an abundance of potential intragenic $\sigma^{54}$ promoters and a number of these promoters were subsequently confirmed experimentally ${ }^{64,65}$. Together, these results suggest that $\sigma^{54}$ promoter elements might indeed be commonly located in intragenic regions.

Many of the natural product $\sigma^{54}$ promoters shown in Figure S1 and listed in Table S3 appear to be internal promoters; promoters located within a gene in an operon instead of upstream of the first gene in the operon. There are examples of internal promoters oriented in the antisense direction relative to the genes in an operon ${ }^{65-68}$; however, this does not seem to be the case for the above internal $\sigma^{54}$ promoters. Instead, each promoter is predicted to yield mRNA corresponding to a subset of an operon's genes. As noted in a recent review, one potential advantage of using internal promoters would be the ability to express only the genes needed in a particular environment ${ }^{69}$.

A second and relatively large group of putative natural product $\sigma^{54}$ promoters is apparent from scanning Figure S1 and Table S3. Namely, many of the putative $\sigma^{54}$ promoters are located in the coding sequence of a gene that is upstream of the single gene or operon they are predicted to regulate, even though the single gene or operon has an upstream and adjacent intergenic region that is long enough to accommodate a $\sigma^{54}$ promoter $\left(\sigma^{54}\right.$-RNA polymerase binding site). The question that arises is, why would a $\sigma^{54}$ promoter be located so far upstream of the single gene or operon whose transcription it aims to regulate? One possibility is that the natural product $\sigma^{54}$ promoter is positioned at a distant location to produce a relatively long $5^{\prime}$ untranslated region (UTR) in the mRNA and that the $5^{\prime}$ UTR provides an additional layer of regulation for natural product gene expression. We should note that a substantial number of the internal $\sigma^{54}$ promoters are predicted to yield relatively long $5^{\prime}$ UTRs as well. Why would many natural product genes need multiple levels of regulation? Natural product synthesis is often energy expensive and many natural products are toxic at high levels; hence, tight and signal-responsive regulation of natural product gene expression might be important in many cases.

Of course, addressing questions about the role and mechanism of $\sigma^{54}$-mediated regulation of natural product genes and other types of bacterial genes will require researchers to go beyond predicting where $\sigma^{54}$ promoters are located. Indeed, we argue that the work of confirming putative $\sigma^{54}$ promoters and analyzing the mRNAs under $\sigma^{54}$ control is crucial, as the results will help address a number of long-held assumptions about bacterial promoters and bacterial gene regulation in general.

Received: 17 November 2020; Accepted: 5 February 2021

Published online: 26 February 2021

\section{References}

1. Barrios, H., Valderrama, B. \& Morett, E. Compilation and analysis of sigma(54)-dependent promoter sequences. Nucleic Acids Res. 27, 4305-4313. https://doi.org/10.1093/nar/27.22.4305 (1999).

2. Feklístov, A., Sharon, B. D., Darst, S. A. \& Gross, C. A. Bacterial sigma factors: A historical, structural, and genomic perspective. Annu. Rev. Microbiol. 68, 357-376. https://doi.org/10.1146/annurev-micro-092412-155737 (2014).

3. Popham, D. L., Szeto, D., Keener, J. \& Kustu, S. Function of a bacterial activator protein that binds to transcriptional enhancers. Science 243, 629-635 (1989).

4. Sasse-Dwight, S. \& Gralia, J. D. Role of eukaryotic-type functional domains found in the prokaryotic enhancer receptor factor б54. Cell 62, 945-954 (1990).

5. Wedel, A. \& Kustu, S. The bacterial enhancer-binding protein NTRC is a molecular machine: ATP hydrolysis is coupled to transcriptional activation. Genes Dev. 9, 2042-2052 (1995).

6. Gao, F. et al. Bacterial enhancer binding proteins-AAA+ proteins in transcription activation. Biomolecules 10, 351 (2020).

7. Bush, M. \& Dixon, R. The role of bacterial enhancer binding proteins as specialized activators of $\sigma 54$-dependent transcription. Microbiol. Mol. Biol. Rev. 76, 497-529 (2012).

8. Su, W., Porter, S., Kustu, S. \& Echols, H. DNA-looping and enhancer activity: association between DNA-bound NtrC activator and RNA polymerase at the bacterial glnA promoter. Proc. Natl. Acad. Sci. 87, 5504-5508 (1990).

9. Carmona, M. \& Magasanik, B. Activation of transcription at $\sigma 54$-dependent promoters on linear templates requires intrinsic or induced bending of the DNA. J. Mol. Biol. 261, 348-356 (1996).

10. Rippe, K., Guthold, M., von Hippel, P. H. \& Bustamante, C. Transcriptional activation via DNA-looping: visualization of intermediates in the activation pathway of E. coli RNA polymerase. $\sigma 54$ holoenzyme by scanning force microscopy. J. Mol. Biol. 270, 125-138 (1997).

11. Shingler, V. Signal sensory systems that impact $\sigma 54$-dependent transcription. FEMS Microbiol. Rev. 35, 425-440 (2011).

12. Magasanik, B. Regulation of transcription of the glnALG operon of Escherichia coli by protein phosphorylation. Biochimie 71, 1005-1012 (1989).

13. Yamada, A., Inoue, T., Noda, S., Hongoh, Y. \& Ohkuma, M. Evolutionary trend of phylogenetic diversity of nitrogen fixation genes in the gut community of wood-feeding termites. Mol. Ecol. 16, 3768-3777 (2007).

14. Hayrapetyan, H., Tempelaars, M., Groot, M. N. \& Abee, T. Bacillus cereus ATCC 14579 RpoN (Sigma 54) is a pleiotropic regulator of growth, carbohydrate metabolism, motility, biofilm formation and toxin production. PLoS ONE 10, e0134872 (2015).

15. Arai, H., Hayashi, M., Kuroi, A., Ishii, M. \& Igarashi, Y. Transcriptional regulation of the flavohemoglobin gene for aerobic nitric oxide detoxification by the second nitric oxide-responsive regulator of Pseudomonas aeruginosa. J. Bacteriol. 187, 3960-3968 (2005).

16. Weiner, L., Brissette, J. \& Model, P. Stress-induced expression of the Escherichia coli phage shock protein operon is dependent on sigma 54 and modulated by positive and negative feedback mechanisms. Genes Dev. 5, 1912-1923 (1991).

17. Anderson, D. K., Ohta, N., Wu, J. \& Newton, A. Regulation of the Caulobacter crescentus rpoN gene and function of the purified $\sigma 54$ in flagellar gene transcription. Mol. Gen. Genet. MGG 246, 697-706 (1995).

18. Arora, S. K., Ritchings, B. W., Almira, E. C., Lory, S. \& Ramphal, R. A transcriptional activator, FleQ, regulates mucin adhesion and flagellar gene expression in Pseudomonas aeruginosa in a cascade manner. J. Bacteriol. 179, 5574-5581 (1997).

19. Jiménez-Fernández, A. et al. Complex interplay between FleQ, cyclic diguanylate and multiple $\sigma$ factors coordinately regulates flagellar motility and biofilm development in Pseudomonas putida. PLoS ONE 11, e0163142 (2016).

20. Correa, N. E., Peng, F. \& Klose, K. E. Roles of the regulatory proteins FlhF and FlhG in the Vibrio cholerae flagellar transcription hierarchy. J. Bacteriol. 187, 6324-6332 (2005).

21. Martinez, R. M., Dharmasena, M. N., Kirn, T. J. \& Taylor, R. K. Characterization of two outer membrane proteins, FlgO and FlgP, that influence Vibrio cholerae motility. J. Bacteriol. 191, 5669-5679 (2009). 
22. Shao, X. et al. RpoN-dependent direct regulation of quorum sensing and the Type VI secretion system in Pseudomonas aeruginosa PAO1. J. Bacteriol. 200 (2018).

23. Matsuyama, B. Y. et al. Mechanistic insights into c-di-GMP-dependent control of the biofilm regulator FleQ from Pseudomonas aeruginosa. Proc. Natl. Acad. Sci. 113, E209-E218 (2016).

24. Lloyd, M. G. et al. Targeting the alternative sigma factor RpoN to combat virulence in Pseudomonas aeruginosa. Sci. Rep. 7, 1-12 (2017).

25. Caberoy, N. B., Welch, R. D., Jakobsen, J. S., Slater, S. C. \& Garza, A. G. Global mutational analysis of NtrC-like activators in Myxococcus xanthus: identifying activator mutants defective for motility and fruiting body development. J. Bacteriol. 185, 6083-6094 (2003).

26. Diodati, M. E. et al. Nla18, a key regulatory protein required for normal growth and development of Myxococcus xanthus. J. Bacteriol. 188, 1733-1743 (2006).

27. Ossa, F. et al. The Myxococcus xanthus Nla4 protein is important for expression of stringent response-associated genes, ppGpp accumulation, and fruiting body development. J. Bacteriol. 189, 8474-8483 (2007).

28. Goldman, B. et al. Evolution of sensory complexity recorded in a myxobacterial genome. Proc. Natl. Acad. Sci. 103, 15200-15205 (2006).

29. Wu, S. S. \& Kaiser, D. Genetic and functional evidence that type IV pili are required for social gliding motility in Myxococcus xanthus. Mol. Microbiol. 18, 547-558 (1995).

30. Giglio, K. M., Caberoy, N., Suen, G., Kaiser, D. \& Garza, A. G. A cascade of coregulating enhancer binding proteins initiates and propagates a multicellular developmental program. Proc. Natl. Acad. Sci. 108, E431-E439 (2011).

31. Kaufman, R. I. \& Nixon, B. T. Use of PCR to isolate genes encoding sigma54-dependent activators from diverse bacteria. J. Bacteriol. 178, 3967-3970 (1996).

32. Gorski, L. \& Kaiser, D. Targeted mutagenesis of $\varsigma 54$ activator proteins in Myxococcus xanthus. J. Bacteriol. 180, 5896-5905 (1998).

33. Guo, D., Wu, Y. \& Kaplan, H. B. Identification and characterization of genes required for early Myxococcus xanthus developmental gene expression. J. Bacteriol. 182, 4564-4571 (2000).

34. Sun, H. \& Shi, W. Genetic studies of mrp, a locus essential for cellular aggregation and sporulation of Myxococcus xanthus. J. Bacteriol. 183, 4786-4795 (2001).

35. Kirby, J. R. \& Zusman, D. R. Chemosensory regulation of developmental gene expression in Myxococcus xanthus. Proc. Natl. Acad. Sci. 100, 2008-2013 (2003).

36. Jelsbak, L. \& Kaiser, D. Regulating pilin expression reveals a threshold for S motility in Myxococcus xanthus. J. Bacteriol. 187, 2105-2112 (2005).

37. Fimlaid, K. A. \& Shen, A. Diverse mechanisms regulate sporulation sigma factor activity in the Firmicutes. Curr. Opin. Microbiol. 24, 88-95 (2015)

38. Stevens, D. C. et al. Alternative sigma factor over-expression enables heterologous expression of a type II polyketide biosynthetic pathway in Escherichia coli. PLoS ONE 8, e64858 (2013).

39. Giglio, K. M. \& Garza, A. G. Fruiting body development in myxococcus xanthus: a multicellular developmental programme that leads to sporulation. Bacterial Spores Curr. Res. Appl. 19 (2012).

40. Volz, C., Kegler, C. \& Müller, R. Enhancer binding proteins act as hetero-oligomers and link secondary metabolite production to myxococcal development, motility, and predation. Chem. Biol. 19, 1447-1459 (2012).

41. Knauber, T. et al. Mutation in the rel gene of Sorangium cellulosum affects morphological and physiological differentiation. Mol. Microbiol. 69, 254-266 (2008).

42. Washio, K., Lim, S. P., Roongsawang, N. \& Morikawa, M. Identification and characterization of the genes responsible for the production of the cyclic lipopeptide arthrofactin by Pseudomonas sp. MIS38. Biosci. Biotechnol. Biochem. 74, 992-999 (2010).

43. Alam, M. T. et al. Metabolic modeling and analysis of the metabolic switch in Streptomyces coelicolor. BMC Genom. 11, 1-9 (2010).

44. Nieselt, K. et al. The dynamic architecture of the metabolic switch in Streptomyces coelicolor. BMC Genom. 11, 1-9 (2010).

45. Shin, H., Youn, J., An, D. \& Cho, K. Production of antimicrobial substances by strains of myxobacteria Corallococcus and Myxococcus. Microbiol. Biotechnol. Lett. 41, 44-51 (2013).

46. Kaiser, D. Social gliding is correlated with the presence of pili in Myxococcus xanthus. Proc. Natl. Acad. Sci. 76, 5952-5956 (1979).

47. Fisseha, M., Gloudemans, M., Gill, R. E. \& Kroos, L. Characterization of the regulatory region of a cell interaction-dependent gene in Myxococcus xanthus. J. Bacteriol. 178, 2539-2550 (1996).

48. Kroos, L., Kuspa, A. \& Kaiser, D. A global analysis of developmentally regulated genes in Myxococcus xanthus. Dev. Biol. 117, 252-266 (1986).

49. Kaplan, H., Kuspa, A. \& Kaiser, D. Suppressors that permit A-signal-independent developmental gene expression in Myxococcus xanthus. J. Bacteriol. 173, 1460-1470 (1991).

50. Studholme, D. J., Buck, M. \& Nixon, T. Identification of potential $\sigma \mathrm{N}$-dependent promoters in bacterial genomes. Microbiology 146, 3021-3023 (2000).

51. Keseler, I. M. \& Kaiser, D. An early A-signal-dependent gene in Myxococcus xanthus has a sigma 54-like promoter. J. Bacteriol. 177, 4638-4644 (1995).

52. Garza, A. G., Harris, B. Z., Greenberg, B. M. \& Singer, M. Control of asgE expression during growth and development of Myxococcus xanthus. J. Bacteriol. 182, 6622-6629 (2000).

53. Giglio, K. M., Zhu, C., Klunder, C., Kummer, S. \& Garza, A. G. The enhancer binding protein Nla6 regulates developmental genes that are important for Myxococcus xanthus sporulation. J. Bacteriol. 197, 1276-1287 (2015).

54. Sarwar, Z. \& Garza, A. G. The Nla28S/Nla28 two-component signal transduction system regulates sporulation in Myxococcus xanthus. J. Bacteriol. 194, 4698-4708 (2012).

55. Sarwar, Z. \& Garza, A. G. Two-component signal transduction systems that regulate the temporal and spatial expression of Myxococcus xanthus sporulation genes. J. Bacteriol. 198, 377-385 (2016).

56. Singer, M. \& Kaiser, D. Ectopic production of guanosine penta-and tetraphosphate can initiate early developmental gene expression in Myxococcus xanthus. Genes Dev. 9, 1633-1644 (1995).

57. Harris, B. Z., Kaiser, D. \& Singer, M. The guanosine nucleotide (p) ppGpp initiates development and A-factor production inMyxococcus xanthus. Genes Dev. 12, 1022-1035 (1998).

58. Cashel, M. The stringent response. Escherichia coli and Salmonella typhimulium: cellular and molecular biology 2, 1458-1496 (1996).

59. Hesketh, A., Sun, J. \& Bibb, M. Induction of ppGpp synthesis in Streptomyces coelicolor A3 (2) grown under conditions of nutritional sufficiency elicits actII-ORF4 transcription and actinorhodin biosynthesis. Mol. Microbiol. 39, 136-144 (2001).

60. Kang, S. G., Jin, W., Bibb, M. \& Lee, K. J. Actinorhodin and undecylprodigiosin production in wild-type and relA mutant strains of Streptomyces coelicolor A3 (2) grown in continuous culture. FEMS Microbiol. Lett. 168, 221-226 (1998).

61. Jacques, P. -É., Rodrigue, S., Gaudreau, L., Goulet, J. \& Brzezinski, R. Detection of prokaryotic promoters from the genomic distribution of hexanucleotide pairs. BMC Bioinformatics 7, 423 (2006).

62. Wang, H. \& Benham, C. J. Promoter prediction and annotation of microbial genomes based on DNA sequence and structural responses to superhelical stress. BMC Bioinformatics 7, 248 (2006).

63. Rangannan, V. \& Bansal, M. Identification and annotation of promoter regions in microbial genome sequences on the basis of DNA stability. J. Biosci. 32, 851-862 (2007). 
64. Bonocora, R. P., Smith, C., Lapierre, P. \& Wade, J. T. Genome-scale mapping of Escherichia coli $\sigma 54$ reveals widespread, conserved intragenic binding. PLoS Genet. 11, el005552 (2015).

65. Bono, A. C. et al. Novel DNA binding and regulatory activities for $\sigma 54(\mathrm{RpoN})$ in Salmonella enterica serovar Typhimurium $14028 \mathrm{~s}$. J. Bacteriol. 199 (2017).

66. Dornenburg, J. E., DeVita, A. M., Palumbo, M. J. \& Wade, J. T. Widespread antisense transcription in Escherichia coli. MBio 1 (2010).

67. Hartkoorn, R. C. et al. Genome-wide definition of the SigF regulon in Mycobacterium tuberculosis. J. Bacteriol. 194, 2001-2009 (2012).

68. Fitzgerald, D. M., Bonocora, R. P. \& Wade, J. T. Comprehensive mapping of the Escherichia coli flagellar regulatory network. PLoS Genet 10, e1004649 (2014).

69. Mejía-Almonte, C. et al. Redefining fundamental concepts of transcription initiation in bacteria. Nat. Rev. Genet. 21, 699-714 (2020).

\section{Acknowledgements}

This work was supported by National Institute of General Medical Sciences grant 1R15GM120714-01 to A.G.G. and R.D.W. We also thank Carlos Castañeda from Syracuse University and Haiyan Li from SUNY Upstate Medical University for access and use of FPLC for protein purification and Bio-Rad imager for EMSAs.

\section{Author contributions}

A.G.G. and M.M. wrote the manuscript. A.G.G., R.D.W. and M.M. edited the manuscript. A.G.G., R.D.W. and M.M. devised the experiments and analyses. M.M. performed all experiments and analyses.

\section{Competing interests}

The authors declare no competing interests.

\section{Additional information}

Supplementary Information The online version contains supplementary material available at https://doi. org/10.1038/s41598-021-84057-4.

Correspondence and requests for materials should be addressed to A.G.G.

Reprints and permissions information is available at www.nature.com/reprints.

Publisher's note Springer Nature remains neutral with regard to jurisdictional claims in published maps and institutional affiliations.

(c) (i) Open Access This article is licensed under a Creative Commons Attribution 4.0 International License, which permits use, sharing, adaptation, distribution and reproduction in any medium or format, as long as you give appropriate credit to the original author(s) and the source, provide a link to the Creative Commons licence, and indicate if changes were made. The images or other third party material in this article are included in the article's Creative Commons licence, unless indicated otherwise in a credit line to the material. If material is not included in the article's Creative Commons licence and your intended use is not permitted by statutory regulation or exceeds the permitted use, you will need to obtain permission directly from the copyright holder. To view a copy of this licence, visit http://creativecommons.org/licenses/by/4.0/.

(C) The Author(s) 2021 